\title{
Profile of atypical-antipsychotics use in patients affected by dementia in the University Hospital of Ferrara
}

\author{
Giacomo Chiabrando • Stefano Bianchi • \\ Elisabetta Poluzzi • Nicola Montanaro • \\ Paola Scanavacca
}

Received: 15 November 2009 / Accepted: 8 April 2010/Published online: 13 May 2010

(C) Springer-Verlag 2010

\begin{abstract}
Background The use of off-label atypical antipsychotic drugs (AA) has been noted for the treatment of behavior disorders in older patients affected by Alzheimer's or by other forms of dementia, even though effectiveness data are limited and use seems to be associated with severe cerebrovascular risks. The data concerning such risks caused the Italian Ministry of Health to release a statement discouraging doctors from prescribing olanzapine and risperidone outside of the registered indications, in May 2004. This study aimed to analyze the prescriptive profile of AAs in patients with dementia, in terms of the choice of active substance and of the clinical characteristics of the patients.

Methods Patients with a diagnosis of dementia and in treatment with AA (risperidone, olanzapine, and quetiapine) were selected from three main Alzheimer Evaluation Centers (Geriatrics, Neurology, Internal Medicine) of the University Hospital in Ferrara, in the period 05/2003-04/ 2006. For each subject, the following information was collected: the frequency of prescriptions, the drug received, and the amount of AAs in the considered period. In the third year of observation, the intensity of treatment was evaluated (intense treatment: $>300$ tablets of any AA; weak
\end{abstract}

G. Chiabrando

S. Orsola University Hospital,

Bologna, Italy

S. Bianchi $(\bowtie) \cdot$ P. Scanavacca

Department of Pharmacy, University Hospital of Ferrara,

Ferrara, Italy

e-mail: farmacia@ospfe.it

E. Poluzzi $\cdot$ N. Montanaro

Department of Pharmacology, University of Bologna,

Bologna, Italy treatment: $<300$ tablets of any AA or up to three packages of risperidone drops). Such data were analyzed in terms of the type of dementia, the behavioral disturbance, and the possible presence of psychomotor agitation. In addition, the adverse reactions that occurred during the treatment were gathered. Lastly, the use of acetylcholinesterase inhibitors among the selected subjects was described.

Results Among the 392 subjects (63\% female), Alzheimer's $(49 \%)$ was the most frequent form of dementia, hallucinations were present in $50 \%$ of the cases and aggression in 53\%.The statement by the Ministry of Health resulted in a foreseeable increase in the consumption of quetiapine and a parallel decrease in risperidone and olanzapine; subsequently, the distribution among the drugs stabilized to similar percentages. The doses used for the control of behavioral disturbances during dementia were on average much lower than those for treating more severe psychoses. Among the patients followed in the third year of observation $(n=159)$, the number of subjects in intense treatment was greater than those in weak treatment (60 vs 40\%). Olanzapine was the AA most frequently used in intense dosages. Among the patients in weak treatment, about $50 \%$ used risperidone, available as oral droplets. In the patients at the Geriatric Center $(n=174)$, in the initial period of analysis 10 adverse events were observed and out of these 10 subjects, all of whom were under intense treatment, 8 out of 10 took quetiapine. The most frequently observed adverse events were tremors, a typical extrapyramidal symptom.

Conclusions As physicians await next studies helping to identify specific classes of drugs for specific symptoms or subpopulations, they should turn to pharmacological treatment only after a careful risk-benefit evaluation. They should consider both the important role of the relationship between patient and carers and the adverse effects of antipsychotics, which are particularly dangerous in the elderly. 
Keywords Behavioral disturbance - Dementia - Atypical antipsychotics $\cdot$ Drug regimen $\cdot$ Adverse drug reactions

\section{Introduction}

Dementia presently represents one of the largest public health problems, mainly as a result of the progressive aging of Western populations [1]. Dementia is defined as a deterioration of the cognitive capacities of the subject for carrying out normal activities of daily life [2]. Estimates of the prevalence of dementia indicate that in western European countries, the number of affected patients will increase by $43 \%$ by the year 2020 compared with 2001 , and by $100 \%$ by the year 2040 . The disorder occurs in $10 \%$ of people in the seventh decade of life and in $20-30 \%$ in the eighth and ninth decades. In institutional contexts, such as retirement homes, it is believed that $60-70 \%$ of individuals are affected by dementia. Alzheimer's disease is the most common cause of dementia (43-64\% of cases) [3, 4].

From a clinical point of view, in addition to Alzheimer's, the other cases are classified as either vascular dementia or mixed dementia. There are also degenerative dementias that are not Alzheimer's and include dementia with Lewy bodies and Pick syndrome, most often diagnosed postmortem during autopsy. By now it seems certain that cerebrovascular disorders are at the root of all types of dementia, including Alzheimer's [5].

Clinical manifestations of dementia can be synthetically distinguished in terms of cognitive symptoms and psychological and behavior disturbances, called BPSD (behavioral and psychological symptoms of dementia). Consequently, a correct therapeutic approach to a patient with dementia must aim at reaching two distinct, but also often correlated, clinical outcomes: better cognitive func- tions and control over psychological and behavioral symptoms. Cognitive symptoms, such as memory loss and language disturbances, are currently treated with cholinesterase inhibitors (donepezil, rivastigmine, galantamine) $[4,6,7]$.

In dementia, BPSD is rather common, above all in the advanced phases of the disease [8]. There is the presence of heterogeneous clinical manifestations that include noncollaborative or destructive behavior symptoms, agitation, incontinence, verbal aggression, noisiness, inappropriate social behavior, sleep disturbances, symptoms of depression, hallucinations, and persecutory delirium [9]. In general, manifestations of delirium are present in $40 \%$ of cases, agitation in $40-90 \%$, and aggressive conduct in $20-40 \%$ [10].

In case of failure of the nonpharmacological environmental approach [11, 12], drug use has to be considered [13]. The use of older antipsychotics has been widely diffused, despite serious side effects (arrhythmia). In particular, low-power agents, such as phenothiazines, are responsible for sedation, orthostasis, and anticholinergic effects, while high-power agents, such as haloperidol, often cause extrapyramidal symptoms (reduction of motor activity, rigidity, or acathisia) and at times also the late onset of dyskinesia, which compromises the mobility of such patients. As has been noted, patients affected by dementia, often elderly, have a low threshold for onset of all of these side effects.

Atypical antipsychotics represent a class of drugs that has been marketed since 1995 and nowadays includes clozapine, olanzapine, risperidone, quetiapine, and aripiprazole. Their use is rarely associated with extrapyramidal symptoms, at least in therapeutic doses, and they seem to control negative symptoms of schizophrenia (apathy, catatony, lack of thoughts). Therefore, atypical antipsychotic drugs have replaced the traditional drugs [15], even
Table 1 Frequency of the different diagnoses of dementia and behavior disturbances in the 392 subjects

\begin{tabular}{llll}
\hline & Description & Number & Percentage \\
\hline Dementia & Alzheimer's & 194 & $49 \%$ \\
& Mixed & 109 & $28 \%$ \\
& Vascular & 42 & $11 \%$ \\
& Parkinson's & 36 & $9 \%$ \\
& Other & 21 & $5 \%$ \\
Psychological/behavioral disturbance & Front-temporal & 11 & $3 \%$ \\
& With Lewy bodies & 7 & $2 \%$ \\
& Restlessness & 315 & $80 \%$ \\
& Aggression & 207 & $53 \%$ \\
& Delirium & 206 & $53 \%$ \\
& Hallucinations & 198 & $51 \%$ \\
& Sundowning & 56 & $14 \%$ \\
\hline
\end{tabular}


Fig. 1 Distribution of patients according to age range and drug received $(n=392)$

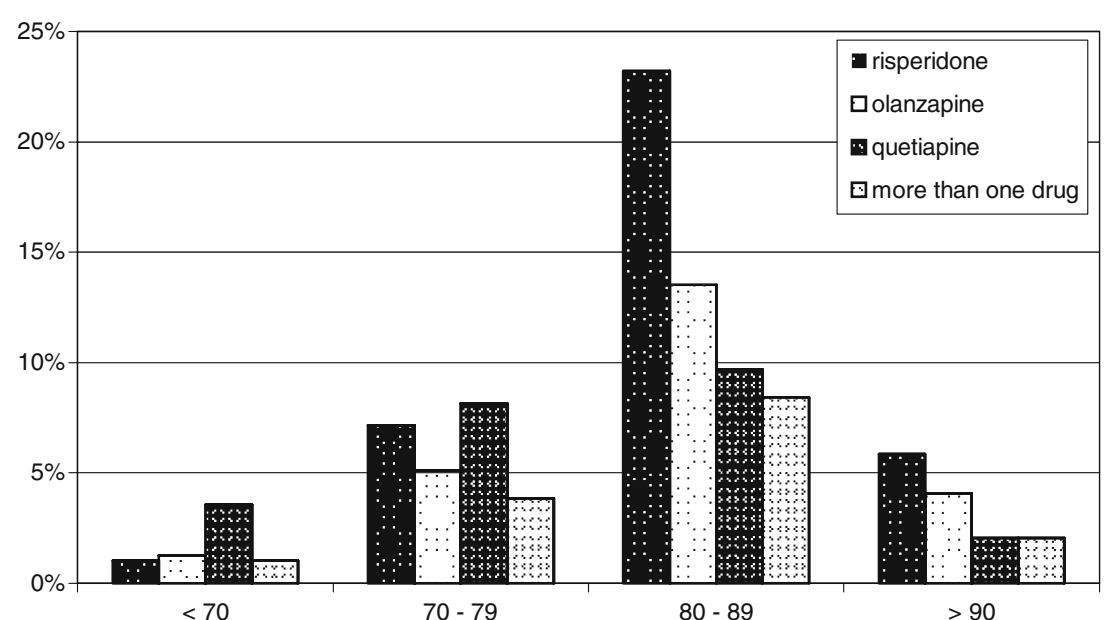

though their use for treating BPSD still remains off-label [14]. Moreover, after some reports of cardiovascular events [16-19] in 2004, many national regulatory agencies published official warnings recommending adherence to label indications [20-22].

The published literature doesn't confirm the greater effectiveness of the atypical antipsychotics. Also in terms of risks it does not seem that the two classes have different profiles [23-25]. Moreover, a review published in 2007 by the Cochrane Library [26] suggested that risperidone and olanzapine are useful in reducing aggression and risperidone is useful in reducing psychosis, but both are associated with the presence of serious cerebrovascular adverse events and extrapyramidal symptoms. In spite of the drugs' modest effectiveness, the significant increase in adverse events confirms that neither risperidone nor olanzapine should be routinely utilized in the treatment of patients with dementia affected by psychosis or aggression, as long as there is not a serious worry or a physical risk for those who work near the patient. A meta-analysis conducted by the FDA, which combined 17 studies of atypical antipsychotics in patients affected by psychological disturbances associated with dementia, showed a significant increase in mortality (OR: 1.7) following the use of atypical antipsychotics compared with placebo [22]. In addition, a meta-analysis from 2005 that considered 15 controlled studies ( 9 not published) found a similar increase in the risk of mortality for atypical antipsychotics compared with placebo $(\mathrm{OR}=$ $1.54,95 \% \mathrm{CI}=0.004-0.02, P=0.01)[27]$.

\section{Aim}

The aim of this study was to evaluate the prescriptive profile of atypical antipsychotics in patients with dementia by analyzing the choice of drugs and the intensity of treatment on the basis of clinical characteristics of the patients.
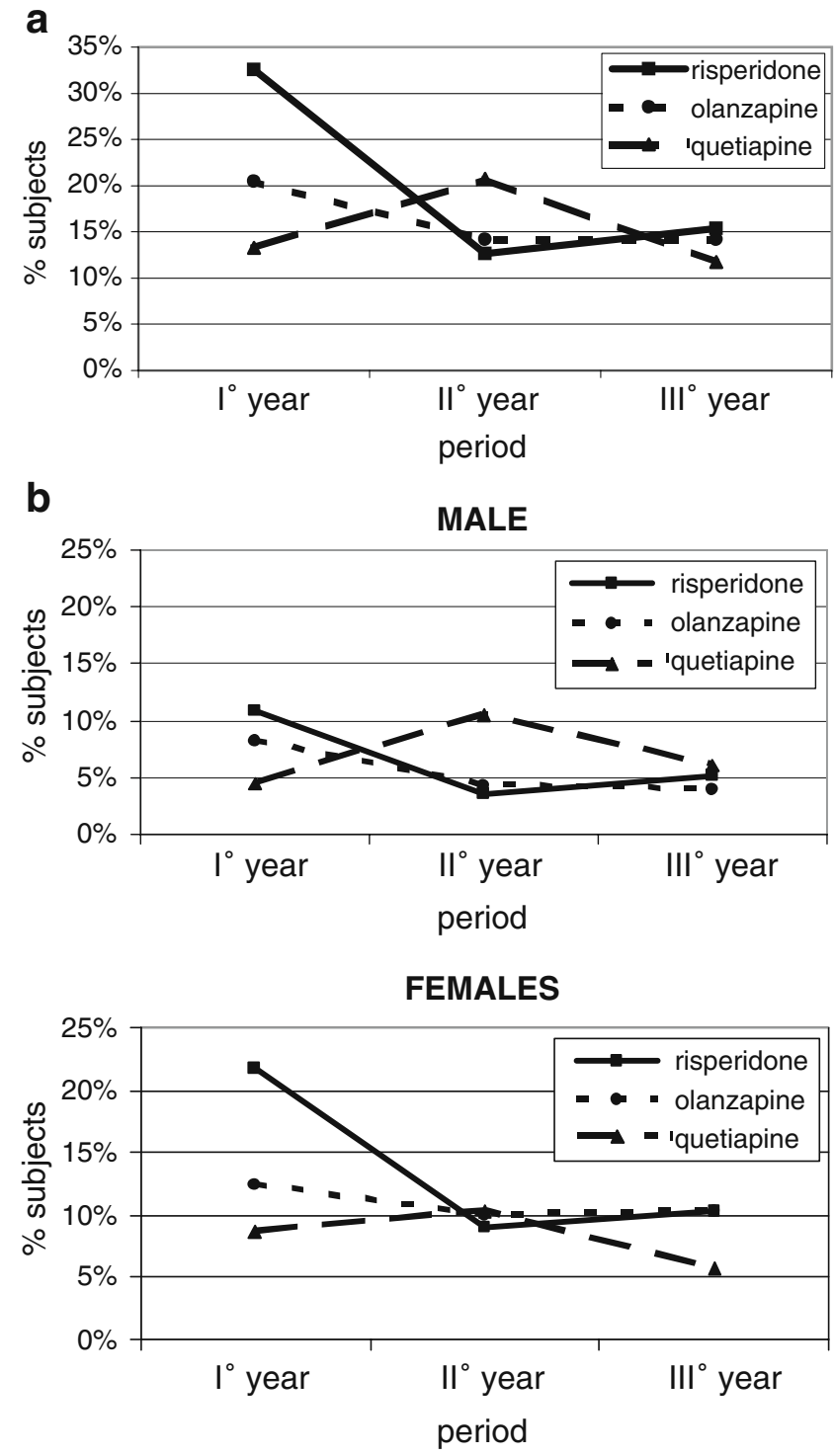

Fig. 2 a Prevalence of yearly use of atypical antipsychotics $(n=332)$. b Prevalence of annual use of atypical antipsychotics in males $(n=126)$ and females $(n=206)$ 


\section{Methods}

Data collection

In Italy, atypical antipsychotics are dispensed to patients affected by dementia only if an ad hoc "treatment plan" to be reviewed each 6 months is completed by the specialist (neurologist, psychiatric or geriatric physician). In addition to the diagnosis of dementia, this plan must contain a diagnosis of behavior disorder. Drugs are supplied by hospital pharmacies after the submission of the treatment plan to the pharmacist.

The following active substances and relevant marketed products are considered in the study: risperidone (Risperdal 60 tablets $1 \mathrm{mg}$, Risperdal 60 tablets $2 \mathrm{mg}$, and Risperdal $100 \mathrm{ml}$ drops $1 \mathrm{mg} / \mathrm{ml}$ ), olanzapine (Zyprexa Velotab 28 tablets $5 \mathrm{mg}$, Zyprexa 28 tablets $5 \mathrm{mg}$, and Zyprexa 28 tablets $2.5 \mathrm{mg}$ ) and quetiapine (Seroquel 60 tablets $25 \mathrm{mg}$, Seroquel 60 tablets $100 \mathrm{mg}$, and Seroquel 60 tablets $100 \mathrm{mg}$ ). Clozapine and aripiprazole were not prescribed in the population observed in our study. For the present study, data on atypical antipsychotics dispensed by the Pharmacy of University Hospital of Ferrara between May 2003 and April 2006 were retrieved. For each dispensed medicinal product, the following information was collected: identification code of the drug, ATC (Anatomical Therapeutic Chemical) code, number of packages and number of DDD (defined daily doses) dispensed, anonymous patient code, patient age and gender, date of prescription, and ward of prescription. Moreover, from the prescription database of the Local Health Authority of Ferrara all prescriptions for acetylcholinesterase inhibitors (donepezil, rivastigmine, and galantamine) supplied between May 2003 and April 2006 were collected.
For the same period of time, all treatment plans for atypical antipsychotic prescriptions submitted to the Pharmacy of the University Hospital of Ferrara were also retrieved, and the following information was collected: anonymous patient code, diagnosis of dementia (i.e., Alzheimer's, fronttemporal, mixed, Parkinson's, Lewy bodies, or vascular) and behavior disorder (i.e., delerium, hallucinations, sundowning, wandering, restlessness, aggression).

The unique patient code allowed the connection to be made between the two different sets of data for individual patients without violating their privacy.

Lastly, adverse drug reactions were collected from the clinical records of the wards.

Study design

We selected all patients affected by dementia and receiving atypical antipsychotic prescriptions in the three Alzheimer Evaluation Centers (Geriatrics, Neurology, and Internal Medicine) at the University Hospital of Ferrara. The prevalence of use in terms of number of subjects receiving at least one prescription in 1 year was evaluated, as well as drug choice, the average daily doses, and the concomitant use of acetylcholinesterase inhibitors, if any. The average daily doses were expressed in milligrams and as a fraction of the DDD. According to Rosholm et al. [28], the average daily dose of each treatment can be calculated as follows:

average daily dose $=$ DDDs $/$ days

where DDDs is the number of DDDs received before the last prescription and days is the number of days between the first and the last prescription. The average daily doses were evaluated for each atypical antipsychotic agent.
Fig. 3 Monthly trend in the patients receiving each antipsychotic $(n=392) . \mathrm{A}=$ warning of the Italian Ministry of Health discouraging the off-label use of AAs

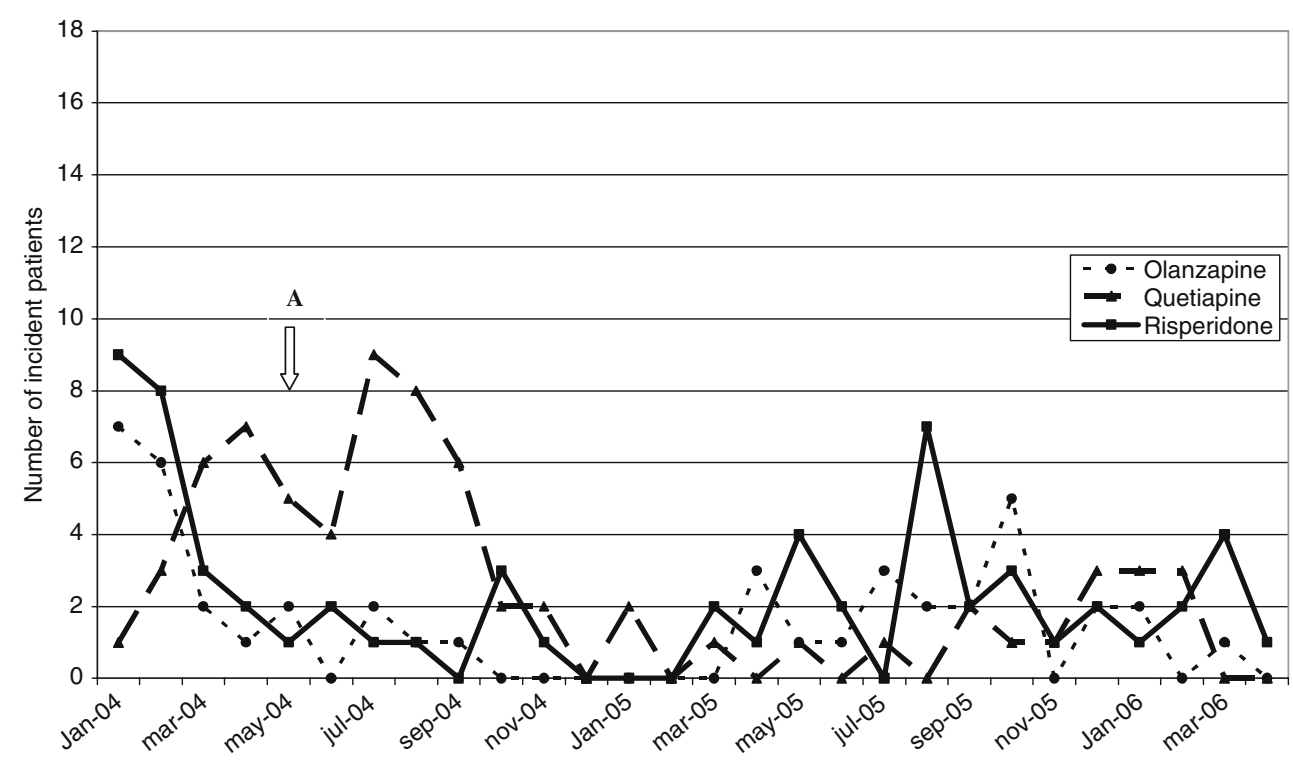




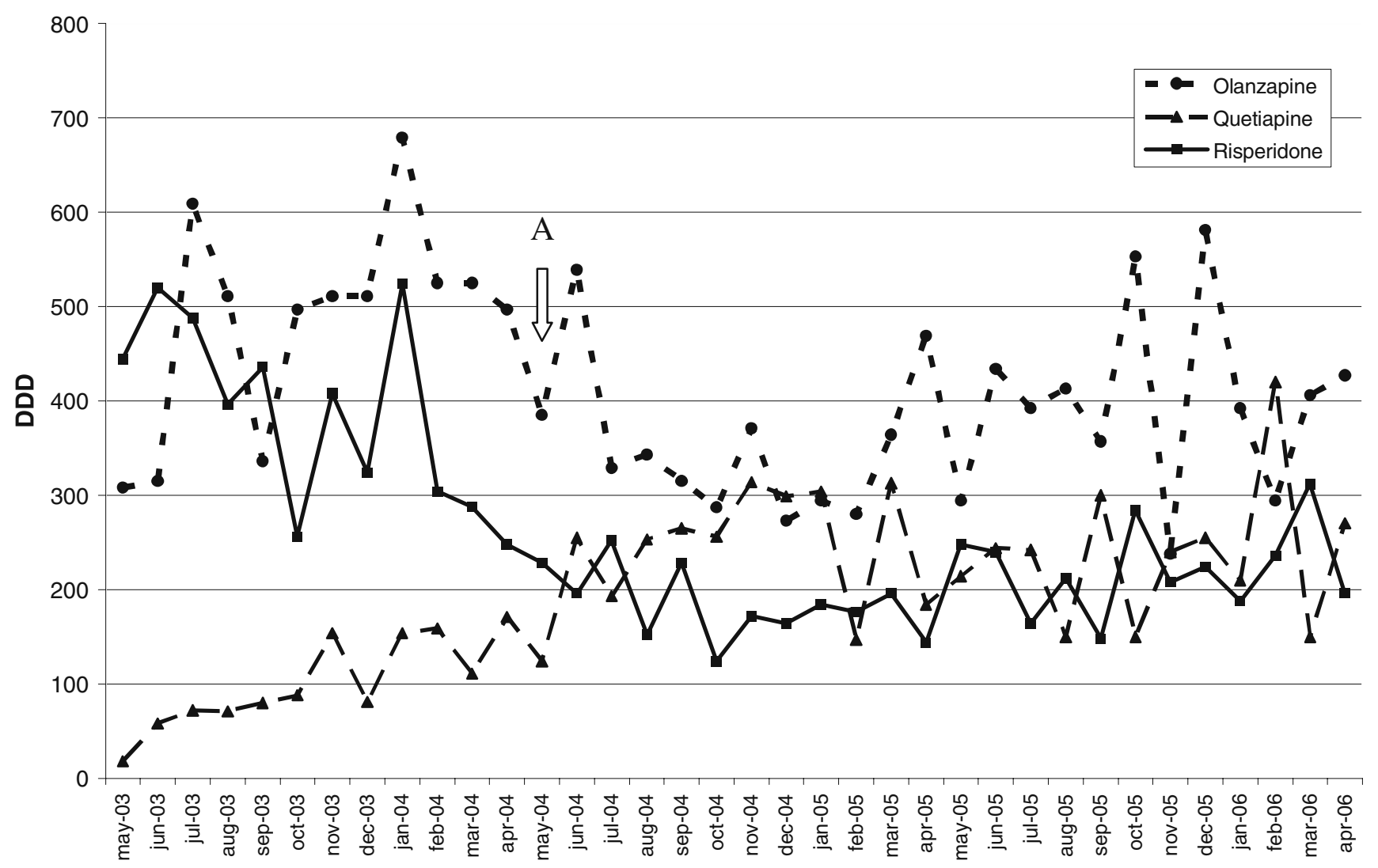

Fig. 4 Monthly trend in drug prescriptions expressed as number of DDDs in patients receiving only one drug $(n=332)$. A= warning of the Italian Ministry of Health discouraging the off-label use of AAs

Using data of the last year of observation $(01 / 05 / 2005-$ $30 / 04 / 2006$ ), the intensity of the drug treatment was evaluated (intense treatment: $>300$ tablets of any atypical antipsychotic; weak treatment: $<300$ tablets of any atypical antipsychotic or up to three packages of risperidone in drops). These data were analyzed according to the type of dementia, the behavior disorder, and the possible presence of psychomotor agitation.

The information was gathered at the Department of Pharmacology at the University of Bologna, in agreement with the Pharmacy of the University Hospital of Ferrara.

\section{Results}

Overall, 2,751 prescription records (456 patients) were retrieved, as well as 793 treatment plans (461 patients), of which 368 were from the Geriatric Unit, 368 from the Neurology Unit, and 57 from Internal Medicine Unit of the University Hospital of Ferrara. Over the 3-year period, $42 \%$ of the patients received more than one treatment plan.

\section{Patient description}

Overall, 392 patients receiving atypical antipsychotics and with diagnosis of dementia were selected (inhabitants of the district of Ferrara: about 159,000).

Women represented 63\% $(n=247)$ of the group of subjects and their ages ranged from 50 to 97 years (the average age was 83 years); men ( $n=145)$ represented $37 \%$ and their ages ranged from 25 to 99 years (the average age was 80 years).

Among the 392 patients, Alzheimer's (49\%) was the most common dementia, followed by mixed (28\%), vascular (11\%), and dementia from Parkinson's disease (9\%). Among
Table 2 Average daily dose expressed as milligrams and as fractions of DDD $(n=332)$

\begin{tabular}{lllll}
\hline ATC V level & $\begin{array}{l}\text { Average daily } \\
\text { dose (mg) }\end{array}$ & $\begin{array}{l}\text { DDD } \\
(\mathrm{mg})\end{array}$ & $\begin{array}{l}\text { Average daily dose } \\
\text { (fraction of DDD) }\end{array}$ & $\begin{array}{l}\text { Average daily dose } \\
\text { recommended in } \\
\text { the literature (mg) }\end{array}$ \\
\hline Olanzapine & 3.5 & 10 & 0.348 & $2.5-5$ \\
Quetiapine & 66 & 400 & 0.165 & $25-100$ \\
Risperidone & 1.08 & 5 & 0.217 & $0.5-2$ \\
\hline
\end{tabular}


Table 3 Use of acetylcholinesterase inhibitors $(n=171)$

\begin{tabular}{lll}
\hline Concomitant treatment & Number & Percentage \\
\hline Donepezil & 94 & $55 \%$ \\
Rivastigmine & 38 & $22 \%$ \\
Galantamine & 14 & $8 \%$ \\
More than one drug & 25 & $15 \%$ \\
Total & 171 & $100 \%$ \\
\hline
\end{tabular}

the behavior disorders, restlessness was almost always present $(80 \%)$, hallucinations were present in $51 \%$ of the cases, while aggression and delirium were found in 53\% (Table 1).

Trend of antipsychotic prescriptions in patients with dementia over a 3-year period

Among the 392 subjects, 94 (24\%) were only prescribed olanzapine, 92 (24\%) only quetiapine, 146 (37\%) only risperidone. Sixty (15\%) were prescribed more than one drug in the 3-year period, of whom eight were prescribed all three. Figure 1 shows the above-mentioned percentages of the patients, stratified according to age.

In patients aged 80 and older, risperidone was prescribed most frequently (42\%), whereas in those aged 70-79, quetiapine was the more prescribed drug (34\%, Fig. 1). Figure $2 \mathrm{a}$ shows the prevalence of use of atypical antipsychotics among the 332 subjects who received only one drug during the 3-year period, no matter the number of prescriptions during the year.

The percentage of subjects receiving risperidone or olanzapine decreased from the first to the second year, and then remained almost constant during the third year (33 vs. 13 vs. $15 \%$ for risperidone, 20 vs. 14 vs. $14 \%$ for olanzapine). At the same time, the percentage of subjects who were prescribed quetiapine increased during the second year of analysis and then decreased during the third year (13 vs. 21 vs. $12 \%)$. The trend in the use of each agent during the 3 -year period was not different between males and females (Fig. 2b), whereas risperidone was more used among females.

Figure 3 illustrates the monthly trend in patients receiving each antipsychotic. After the warning by the Italian Ministry of Health in May 2004 discouraging the off-label use of atypical antipsychotics [21] (indicated by A in Figs. 3 and 4), there was a foreseeable increase in the consumption of quetiapine (the only atypical antipsychotic labeled for generic psychotic disorders) to the detriment of the other two drugs, and subsequently there was a stabilization to similar percentages for the three different drugs (see Fig. 4).

Table 2 shows the average daily doses for each drug.

Among the 392 selected subjects, 171 (47\%) also used acetylcholinesterase inhibitors (Table 3). Donepezil was most commonly prescribed $(55 \%)$, followed by rivastigmine $(22 \%)$. Memantine was not reimbursed by Italian Health Service until 2009.

Yearly pattern of use of antipsychotics in patients with dementia

Among the 392 subjects, patients with a diagnosis of dementia and in treatment with atypical antipsychotics during the third year of observation (May 2005-April 2006) were selected $(n=159)$. Of these, 54 were prescribed risperidone (34\%), 60 olanzapine (38\%), and 45 quetiapine (28\%).

Figure 5 illustrates the frequency of patterns of behavior disturbance diagnoses. For most patients, more than one disturbance was indicated. The most frequent patterns were represented by (1) restlessness concomitantly with aggression (IG, 16\%), (2) delirium, agitation, and restlessness together with aggression (DAIG, 13\%) and (3) delirium, agitation, and restlessness (DAI, 12\%).

Figure 6 illustrates the distribution of patients by behavior disturbance and drugs received. Olanzapine was the more prescribed drug, except for patients suffering from sundowning and wandering, in which situations quetiapine use was the highest.
Fig. 5 Patterns of behavior disturbances $(n=159)$. $D$ Delirium, $I$ restlessness, $A$ agitation, $G$ aggression, $S$ sundowning, $W$ wandering
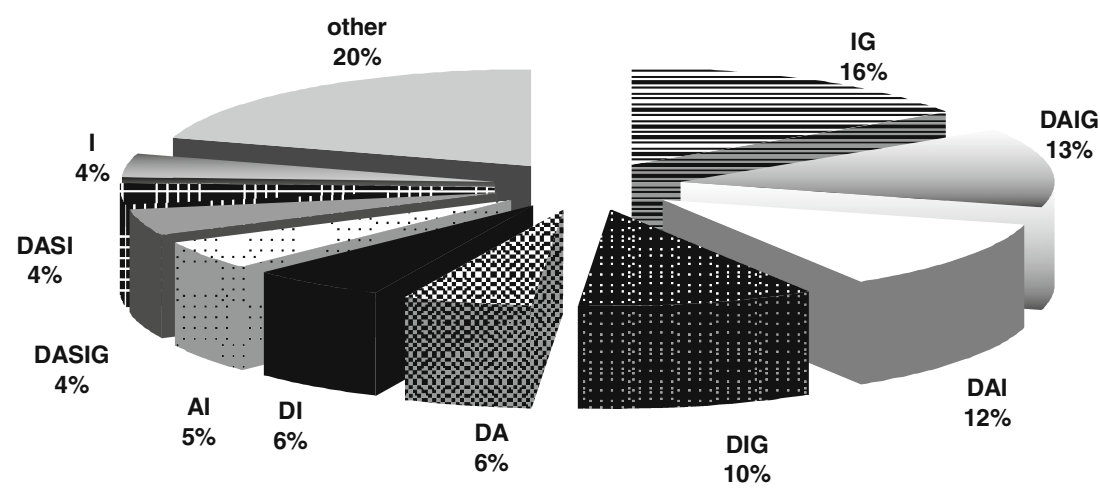


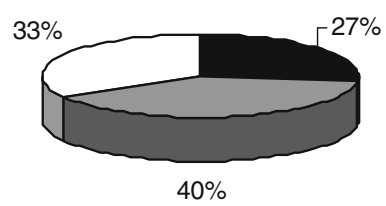

hallucinations

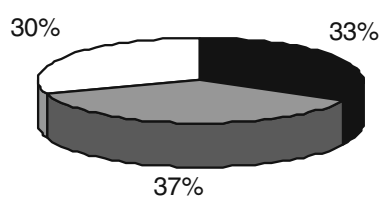

restlessness

sundowning

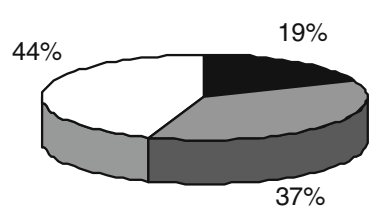

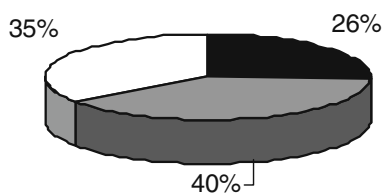

delirium
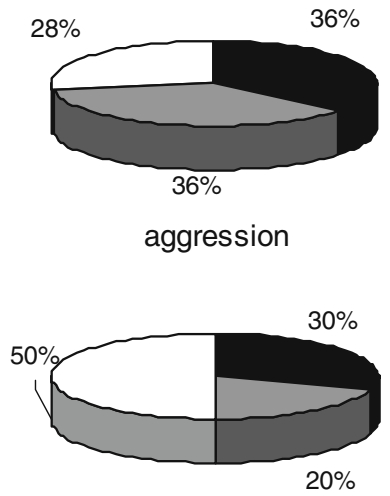

wandering risperidone; $\square$ olanzapine; $\square$ quetiapine

Fig. 6 Frequency of use of atypical antipsychotics for behavior disturbances $(n=159)$

Only one out of three centers (Geriatrics) provided counseling to patients with dementia and behavior disturbance (76 patients, 19\%). Generally, these patients were supported by counseling before drug therapy, whereas in cases of hallucination and delirium, the first choice of treatment was the atypical antipsychotic drug. Figure 7 shows the percentage of patients in treatment with antipsychotics based on the type of dementia.

Among subjects with diagnosis of Alzheimer's (43\%), $41 \%$ were prescribed olanzapine, $35 \%$ risperidone, and
$25 \%$ quetiapine. Mixed dementia was diagnosed in $21 \%$ of the subjects, and among these, olanzapine and quetiapine were prescribed in 35 and 38\%, respectively. Among the subjects with vascular dementia $(7 \%)$, risperidone was the most used drug, while among those with Parkinson's, it was quetiapine $(53 \%)$.

The number of patients in weak treatment was higher than that of patients in intense treatment (61 vs. 39\%). Olanzapine was the most commonly used atypical antipsychotic at intense dosages. Among the patients in weak treatment, half of these used risperidone, a drug taken in drops (result not showed). Patients with more than one behavior disturbance used intense treatments more frequently than others (47\%), whereas those with delirium used them only in $17 \%$ of cases (Table 4 ).

\section{Adverse events}

In the initial period of analysis (05/2003-09/2004), among patients admitted to the Geriatric Center $(n=174), 10$ adverse events were observed, and out of these subjects, regardless of the type of intense treatment, 8 were taking quetiapine. The adverse event most commonly observed was tremors, a typical extrapyramidal effect. In the patients admitted to the Neurological and Internal Medicine Centers, adverse effects were not observed. Figure 8 shows the adverse effects observed in patients in the Geriatric Center.

\section{Discussion}

Among the 392 patients analyzed, Alzheimer's and restlessness represented, respectively, the most common diagnoses of dementia and behavior disturbance. However, the
Fig. 7 Use of atypical antipsychotics by type of dementia $(n=159)$

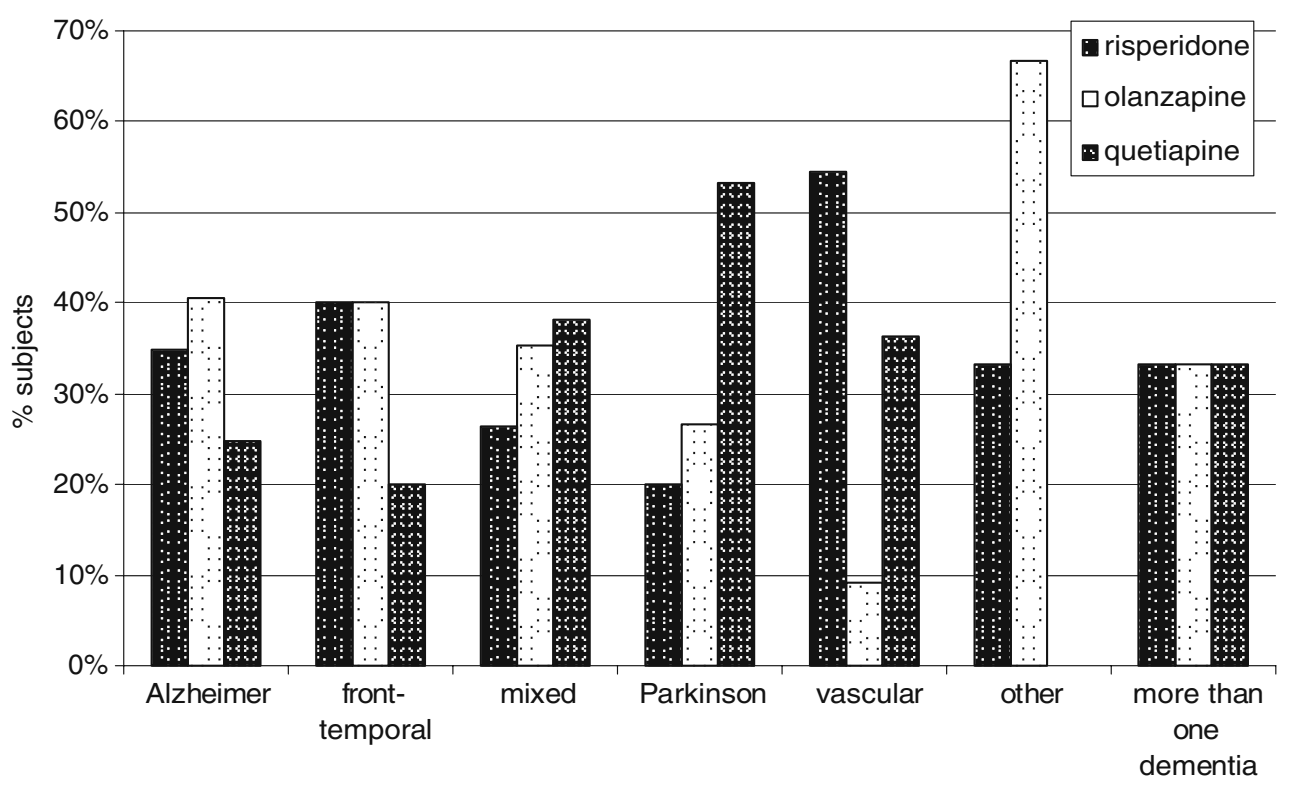


Table 4 Distribution of patients according to the intensity of treatment and behavior disturbance

\begin{tabular}{lllll}
\hline & Intense & Weak & Total & Percent intense \\
\hline Hallucinations & 4 & 8 & 12 & 33 \\
Delirium & 2 & 10 & 12 & 17 \\
Aggression & 8 & 18 & 26 & 31 \\
More than one & 46 & 51 & 97 & 47 \\
$\quad$ disturbance & 2 & 10 & 12 & 17 \\
Other & 62 & 97 & 159 & 39 \\
Total & & &
\end{tabular}

majority of patients presented more than one behavior disturbance.

Over the 3-year period of analysis, risperidone was the most prescribed atypical antipsychotic (37\%) among those receiving monotherapy, while $15 \%$ of subjects received more than one drug. Almost half of the patients were also being treated with acetylcholinesterase inhibitors, in particular donepezil (55\% of cases).

Published literature [29] and clinical practice have clearly demonstrated the need to optimize the treatment of cognitive disorders, either with memantine and acetylcholinesterase inhibitors, before deciding to start antipsychotic therapy. In some patients, antidementia agents can also improve agitation symptoms. Our data did not allow us to evaluate the improvement in behavior disturbance owing to antidementia therapy.

The warning released by the Italian Ministry of Health in May 2004 [21], discouraging the off-label use of atypical antipsychotics, and the numerous relative warnings concerning the safety of these drugs have resulted in a foreseeable increase in the consumption of quetiapine, to the detriment of the other two drugs, in terms of both number of subjects and amount of drug prescribed. Nevertheless, such an effect was resolved in the early

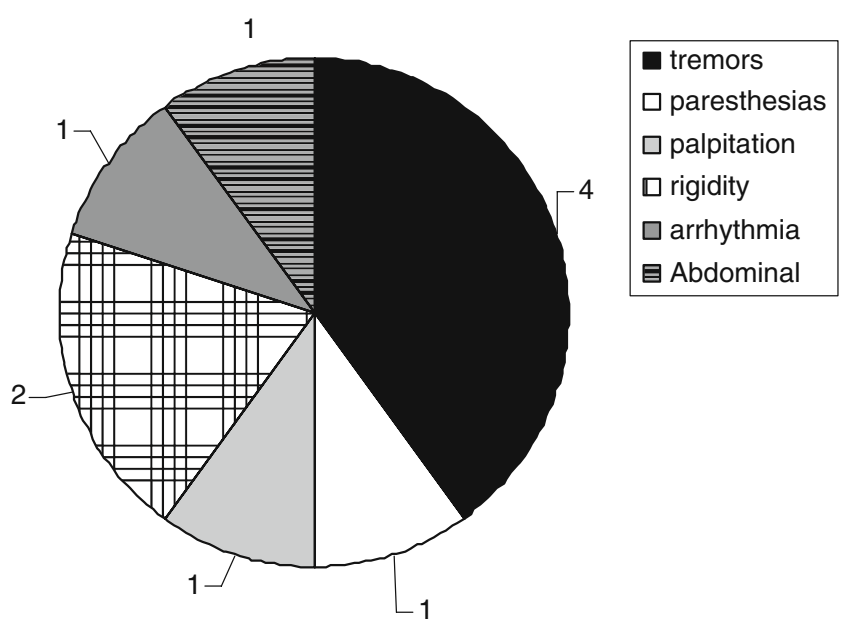

Fig. 8 Number of adverse events observed in dementia patients receiving atypical antipsychotics in the Geriatric Center during the period 05/2003-09/2004 $(n=174)$ months of the application of the measure, subsequently stabilizing the choice of drug and reaching similar percentages for the three different drugs.

The daily doses used to control behavior disturbances during dementia were in agreement with the dosages reported in main published research $[14,25]$ and were very much lower than those used for the treatment of more serious psychoses.

The adverse event most commonly observed was tremor, a typical extrapyramidal effect. This fact shows that it is not only classic antipsychotics that worsen these adverse effects, but also atypical antipsychotics, even if probably with a lower frequency. The absence of cerebrovascular events among those patients observed in the present study is not surprising due to the limited number of patients.

Concerning the pattern of use, the type of behavior disturbance did not largely condition the choice of the atypical antipsychotic, even if olanzapine seemed almost always to be the most used drug, except in cases of sundowning and wandering, where quetiapine is preferred.

When the data regarding utilization are analyzed by type of dementia, olanzapine is the most used drug in cases of Alzheimer's, while in Parkinson's and in vascular dementia, quetiapine and risperidone are preferred, respectively.

Among the 159 patients, the frequency of behavior disorders of this cohort is similar to that of total population of the study. In addition, the number of patients in weak treatment was higher than those in intense treatment. Olanzapine was the antipsychotic most commonly used in intense dosages, above all in the presence of Alzheimer's, compared with risperidone, which is generally used for weak treatment, probably because it is taken in droplets.

The efficacy of psychosocial care in the improvement of behavior disturbance in patients with dementia is well documented. In particular, this approach seems to represent a very effective alternative to neuroleptic drugs especially in case of severe dementia [30]. Nonpharmacological therapy should also be considered in the light of the dubious risk-benefit profile of neuroleptic drugs in patients with dementia [31]. Our data did not allow us to provide a detailed report of nonpharmacological care, but in Italian clinical practice this kind of care seems to be addressed only to 
a limited percentage of patients, probably because of the lack of resources for this aim.

The study results have to be considered in the light of some limitations: in particular, no control group was available, e.g., patients affected by behavior disorders and treated with only nonpharmacological methods, to evaluate the frequency of drug use among patients with these disorders.

In conclusion, future studies should help to identify specific classes of drugs for specific symptoms and for subpopulations of patients. Nowadays, the general practitioner should keep in mind the elevated percentage of response to placebo, which suggests that behavior disturbances or psychoses in patients affected by dementia could be managed with an increase in attention to the patient (identification and management of comorbidity, frequent visits, increase in relationship with the staff). Currently in cases in which it is necessary to turn to a pharmacological treatment, the atypical antipsychotics probably remain the drug of choice, but the general practitioner prescriber and the family must be alerted to the possible risk of these drugs [32].

\section{References}

1. Ott A, Breteler M, van Harskamp F (1995) Prevalence of Alzheimer's disease and vascular dementia: association with education: the Rotterdam study. Br Med J 310:970-973

2. Bird TD, Miller BL. Alzheimer's disease and other dementias. In: Kasper DL, Braunwald E, Fauci AS, et al (eds) Harrison's Internal Medicine. McGraw Hill, New York

3. Morgan K, Lilley JM, Arie T (1993) Incidence of dementia in a representative British sample. Br J Psychiatry 163:467-470

4. AIFA Ministero della Salute (2006) Alzheimer e inibitori delle colinesterasi: c'è qualcosa di nuovo? Boll d'Inf Farm 13(1):19-25

5. Wolfson C, Wolfson DB, Asgharian M et al (2001) A reevaluation of the duration of survival after the onset of dementia. N Engl $\mathrm{J}$ Med 344:1111-1116

6. NHS National Institute for Health and Clinical Excellence (2007) NICE technology appraisal guidance 111. Donepezil, galantamine, rivastigmine (review) and memantine for the treatment of Alzheimer's disease. NHS, London

7. Wolfson C, Oremus M, Shukla V et al (2002) Donepezil and rivastigmine in the treatment of Alzheimer's disease: a best evidence synthesis of the published data on their efficacy and cost-effectiveness. Clin Ther 24:862-886

8. Beck C, Frank L, Chumbler NR (1998) Correlates of disruptive behavior in severely cognitively impaired nursing home residents. Gerontologist 38:189-198

9. Rovner BW (1992) Behavioral and psychiatric disability in Alzheimer's disease. J Geriatr Psychiatry 25:247-255

10. Merriam AE, Aronson MK, Gasten P (1988) The psychiatric symptoms of Alzheimer's disease. J Am Geriatr Soc 36:7-12

11. Forbes DA, Peacock S, Morgan D (2005) Non-pharmacological management of agitated behaviors associated with dementia. Geriatrics Aging 8:26-30

12. Yeager BF, Farnett LE, Ruzicka SA (1995) Management of the behavior manifestations of dementia. Arch Intern Med 155:250-260
13. Sink KM, Holden KF, Yaffe K (2005) Pharmacological treatment of neuropsychiatric symptoms of dementia: a review of the evidence. JAMA 293:596-608

14. Lee PE, Gill SS, Freedman M, Bronskill SE, Hillmer MP, Rochon PA (2004) Atypical antipsychotic drugs in the treatment of behavior and psychological symptoms of dementia: systematic review. BMJ 329:75-79

15. Trifirò G, Spina E, Brignoli $O$ et al (2005) Antipsychotic prescribing pattern among Italian general practitioners: a population based study during the years 1999-2002. Eur J Clin Pharmacol 61:47-53

16. Wooltorton E (2004) Olanzapine: increased incidence of cerebrovascular events in dementia trials. CMAJ 170:1395

17. Wooltorton E (2002) Risperidone: increased rates of cerebrovascular events in dementia trials. CMAJ 167:1269

18. AIFA Ministero della Salute (2004) Importanti informazioni di sicurezza su risperidone e rischio di eventi cerebrovascolari nel trattamento delle psicosi e dei disturbi comportamentali in pazienti anziani affetti da demenza. Boll d'Inf Farm 1:14

19. AIFA Ministero della Salute (2004) Importante informazione di sicurezza su olanzapina e rischio di mortalità e ictus nel trattamento delle psicosi e dei disturbi comportamentali in pazienti anziani affetti da demenza. Boll Inf Farm 1:15

20. Committee on Safety of Medicines (2004) Atypical antipsychotic drugs and stroke. http://www.mhra.gov.uk/Safetyinformation/ Safetywarningsalertsandrecalls/Safetywarningsandmessagesfor medicines/CON1004298

21. Ministero della Salute (2004) Chiarimenti riguardanti la prescrivibilità dei farmaci antipsicotici atipici da parte delle UVA. Farmacovigilanza News 7-8:4

22. FDA (2005) Public health advisory: deaths with antipsychotics in elderly patients with behavioral disturbances. http://www.fda.gov/ Drugs/DrugSafety/PublicHealthAdvisories/UCM053171

23. Wang PS, Schneeweiss S, Avorn J et al (2005) Risk of death in elderly users of conventional vs. atypical antipsychotic medications. N Engl J Med 353:2335-2341

24. Trifirò G, Verhamme KM, Ziere G, Caputi AP, Ch Stricker BH, Sturkenboom MC (2006) All-cause mortality associated with atypical and typical antipsychotics in demented outpatients. Pharmacoepidemiol Drug Saf 16:538-544

25. Schneider LS, Tariot PN, Dagerman KS et al (2006) Effectiveness of atypical antipsychotic drugs in patients with Alzheimer's disease. N Engl J Med 12:1525-1538

26. Ballard C, Waite J, Birks J (2006) Atypical antipsychotics for aggression and psychosis in Alzheimer's disease. Cochrane Database Syst Rev 1:CD003476

27. Schneider LS, Dagerman KS, Insel P (2005) Risk of death with atypical antipsychotic drug treatment. J Am Med Assoc 294 (15):1934-1943

28. Rosholm JU, Gram LF, Isacsson G, Hallas J, Bergman U (1997) Changes in the pattern of antidepressant use upon the introduction of the new antidepressants: a prescription database study. Eur J Clin Pharmacol 52:205-209

29. Daiello LA (2007) Atypical antipsychotics for the treatment of dementia-related behaviors: an update. Med Health R I 90:191194

30. Fossey J, Ballard C, Juszczak E et al (2006) Effects of enhanced psychosocial care on antipsychotic use in nursing home residents with severe dementia: cluster randomized trial. BMJ 332:756-761

31. Schneider L, Dagerman K, Insel P (2006) Efficacy and adverse effects of atypical antipsychotics for dementia: meta-analysis of randomized, placebo-controlled trials. Am J Geriatr Psychiatry 14 (3):191-210

32. Mulsant BH (2005) Review: pharmacotherapy provides minimal improvements in the neuropsychiatric symptoms of dementia. Evid Based Mental Health 8(3):83 